\title{
Serum Irisin Levels in Patients with Acute Atrial Fibrillation
}

\author{
VILDAN OZER ${ }^{1}$, YUNUS KARACA ${ }^{2}$, MUCAHIT GUNAYDIN ${ }^{3}$, SAVAS OZER $^{4}$, MURSEL SAHIN ${ }^{5}, S^{-}$ \\ LEYMAN CANER KARAHAN ${ }^{6}$, ELIF SAHIN ${ }^{7}$, AYNUR SAHIN ${ }^{2}$, ABDULKADIR GUNDUZ ${ }^{8}$ \\ 1 Specialist, Rize State Hospital, Department of Emergency Medicine, Rize, Turkey \\ 2 Assist Prof., Karadeniz Technical University Faculty of Medicine Department of Emergency Medicine, Trabzon, Turkey \\ 3 Assist Prof., Giresun University Faculty of Medicine Department of Emergency Medicine, Giresun, Turkey \\ ${ }_{4}$ Specialist, Recep Tayyip Erdoğan University Faculty of Medicine Department of Cardiology, Rize, Turkey \\ ${ }_{5}$ Assist Prof., Karadeniz Technical University Faculty of Medicine Department of Cardiology, Trabzon, Turkey \\ ${ }_{6}$ Prof., Karadeniz Technical University Faculty of Medicine Department of Biochemistry, Trabzon, Turkey \\ 7 Assistant, Karadeniz Technical University Faculty of Medicine Department of Biochemistry, Trabzon, Turkey \\ s Prof., Karadeniz Technical University Faculty of Medicine Department of Emergency Medicine, Trabzon, Turkey
}

*This study was produced from a doctoral thesis prepared by the first author under the supervision of the second author

Corresponding author:

Mucahit Gunaydin, MD, Assistant Professor

Giresun University Faculty of Medicine, Department of Emergency Medicine, Giresun, Turkey

Postal Address: Giresun Üniversitesi Tip Fakültesi, Gazipaşa Yerleşkesi, Debboy Mevkii, 28100, Giresun, Turkey

Tel: +904543101600

Fax: +904543101699

e-mail:mgunaydin@hotmail.com

\section{ABSTRACT}

Objective. The purpose of this research was to investigate whether changes in serum irisin levels can represent a marker of altered energy requirements in patients with acute atrial fibrillation (AF) undergoing cardioversion (CV).

Methods. The research was planned as a randomized, prospective case-control study. Patients presenting to the emergency medicine and cardiology departments of a university hospital due to acute AF were included in the study. Irisin levels were measured from serum specimens collected 24 and 72 hours (h) following restoration of sinus rhythm with $\mathrm{CV}$ in patients in AF rhythm. The values obtained were then compared using statistical analysis.

Results. Thirty-one patients undergoing $\mathrm{CV}$ due to acute AF were enrolled. Mean irisin levels were studied from serum specimens collected 24 and $72 \mathrm{~h}$ following restoration of sinus rhythm with $\mathrm{CV}$, and were then compared. No statistically significant difference was determined at comparison of patients' basal to $24 \mathrm{~h}$, basal to $72 \mathrm{~h}$, and 24 to $72 \mathrm{~h}$ mean irisin values $(\mathrm{p}=0.734$, $\mathrm{p}=0.958$, and $\mathrm{p}=0.643$, respectively). Negative correlation was determined between basal serum irisin levels and LDL ( $r=-$ $0.519, \mathrm{p}=0.002$ ), but no significant correlation was observed with epicardial adipose tissue (EAT) thickness.

Conclusion. We determined no change in serum irisin levels studied $24 \mathrm{~h}$ and $72 \mathrm{~h}$ following return of normal sinus rhythm after CV from basal serum irisin levels in patients with acute AF. No correlation also was determined between serum irisin levels and EAT thickness.

Key words: Atrial fibrillation, Irisin, Epicardial adipose tissue, Cardioversion

\section{INTRODUCTION}

Atrial fibrillation (AF) is the most common form of cardiac arrhythmia both worldwide and in Turkey and is seen in $1-2 \%$ of the global population (1). Whether or not the body's energy requirement has increased, is important in terms of treatment planning in patients with AF and of explaining the body's response to this form of arrhythmia. Some studies of energy metabolism in patients with AF have reported that the AF rhythm increases energy demand (2-5), while others have reported impaired energy production or consumption (6-9). These inconsistent reports from the literature permit no definite conclusion regarding whether or not energy demand increases in the AF rhythm.

Irisin functions as an adenosine triphosphate (ATP) sensor in local tissues. If there is no energy requirement in the tissue, energy present in physiological irisin lev- els is converted into heat, while if the tissue requires ATP, then irisin production decreases. A low irisin level is thought to bestow some degree of protection against necrosis by inhibiting conversion of ATP into heat (10-12).

In order to investigate the relation between AF and energy metabolism, we planned to determine whether basal serum irisin levels in patients with AF would change 24 and 72 hours (h) after cardioversion (CV).

\section{MATERIALS AND METHODS}

\section{Study Population}

This single-center, prospective study was performed in the emergency medicine and cardiology departments of a Turkish university hospital. Following receipt of ethical committee approval (No. 2016/32), the study was conducted over an eight-month period between April and December, 2016. Patients undergoing medical or electrical $\mathrm{CV}$ procedures due to $\mathrm{AF}$ and in whom sinus rhythm was restored were included in the study. Patients with moderate or more severe heart valve disease, heart valve prosthesis, congenital heart disease, hypertrophic cardiomyopathy, permanent pacemaker, an ejection fraction lower than $50 \%$, or with uncontrolled or untreated thyroid function disorder, pregnant pa- 
tients and patients with kidney failure (creatinine $>1.2 \mathrm{mg} / \mathrm{dl}$ ), chronic liver disease, or diabetes, or aged under 18 , were excluded.

One hundred forty-nine patients undergoing $\mathrm{CV}$ due to $\mathrm{AF}$ were evaluated. One hundred eighteen of these patients were excluded for various reasons. The study was thus finally conducted with 31 patients. The patient selection scheme is shown in Figure 1. Selection of medical or electrical CV was based on European Society of Cardiology (ESC) 2016 guideline recommendations (1). All the patients included in the study were hemodynamically stable, and medical or elective electrical $\mathrm{CV}$ was performed. No emergency electrical CV was performed in any case.

All patients' demographic characteristics, echocardiography (ECHO) findings, body mass index (BMI) values and CHA2DS2VASc scores at time of presentation were recorded onto study forms. Routine biochemical parameters including glucose, creatinine, C-reactive protein (CRP), white blood cell count and platelet count obtained by measuring hemoglobin, thyroid stimulating hormone (TSH), free thyroxine (fT4), low-density lipoprotein (LDL), high-density lipoprotein (HDL), and total cholesterol and triglyceride levels were also recorded.

\section{Echocardiographic Evaluation}

Transthoracic ECHO was performed at time of presentation on all patients with AF. Patients' echocardiographic measurements were obtained using a Vivid 7 (GE Vingmed Ultrasound, Horten, Norway) ECHO device. Left atrium (LA) diameter, left ventricular end-systolic diameter (LVESD) and end-diastolic diameter (LVEDD), and interventricular septum (IVS) and posterior wall (PW) thicknesses from parasternal long axis images together with epicardial adipose tissue (EAT) thickness were measured. Ejection fraction was calculated from apical four chamber view using the modified Simpson method. Right ventricle (RV) and right atrium (RA) diameters were measured. Pulmonary artery pressure (PAP) was measured with continuous Doppler flow from the tricuspid valve jet.

\section{Serum Irisin Measurement}

Two milliliters of serum was separated from blood collected from patients diagnosed with acute AF and placed into biochemistry tubes at time of presenta-

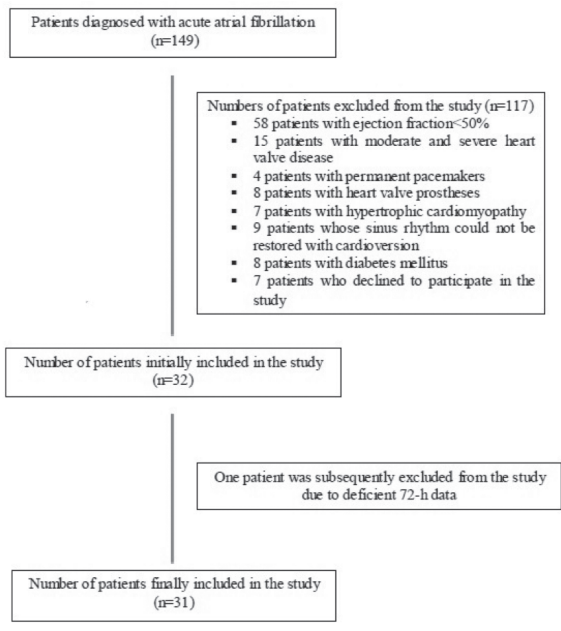

Figure 1. Patient selection scheme

tion and during routine tests after $24 \mathrm{~h}$ and $72 \mathrm{~h}$. The serum specimens collected were stored at $-80{ }^{\circ} \mathrm{C}$ until the study was completed. Irisin levels were determined using an Enzyme-Linked Immunosorbent Assay (ELISA) kit (Elabscience, Cat No. RAG018R, Lot: X17-001, Czech Republic) in line with the manufacturing company's instructions. Serum specimens in storage at $-80{ }^{\circ} \mathrm{C}$ were brought to room temperature. Irisin standards were prepared in line with the kit procedures. Specimen absorbances were read at a $450 \mathrm{~nm}$ wavelength on a VERSA (Designed by Molecular Devices in California, USA) microplate reader. The results were expressed as $\mu \mathrm{g} / \mathrm{mL}$. Other biochemical parameters (glucose, creatinine, cholesterol, etc.) were measured using standard laboratory methods.

\section{Statistical Analysis}

Data analysis was performed on SPSS 23.0 statistical software. Descriptive statistics were expressed as number and percentage for categorical variables and as mean plus standard deviation for numerical variables. Normal distribution of numerical variables was assessed using the KolmogorovSmirnov test. The paired t test was used to compare normally distributed dependent variables. At correlation analysis, Pearson's correlation test was used for normally distributed data, and Spearman's correlation test for non-normally distributed data. Statistical significance was set at $\mathrm{p}<0.05$.

\section{RESULTS}

Patients' mean age was $59.1 \pm 10.9$ years; 17 (54.8\%) were women and $14(45.2 \%)$ were men. Paroxysmal AF was present in $21(67.7 \%)$ patients and persistent AF in $10(32.3 \%)$. Fourteen $(45.2 \%)$ patients received medical CV and 17 (54.8\%) electrical CV. The demographic characteristics of the patient groups are shown in Table 1. Patients' basal biochemical data and serum irisin values are shown in Table 2. Initial serum irisin levels measured with patients in AF rhythm were $7.68 \pm 2.96 \mu \mathrm{g} / \mathrm{mL}$, while 24 -h values were $7.52 \pm 2.56 \mu \mathrm{g} / \mathrm{mL}$, and $72-\mathrm{h}$ values were $7.71 \pm 2.63 \mu \mathrm{g} / \mathrm{mL}$. No statistically significant difference was determined between basal and 24-n, basal and $72-\mathrm{h}$, or $24-\mathrm{h}$ and $72-\mathrm{h}$ serum irisin values $(\mathrm{p}=0.734, \mathrm{p}=0.958$, and $\mathrm{p}=0.643$ ).

Correlations between patients' basal serum irisin levels and clinical characteristics, ECHO findings, and laboratory findings are shown in Table 3. Significant negative correlation was determined only between basal serum irisin levels and the lipid parameter LDL $(r=-0.519, p=0.002)$. No significant correlation was determined between basal serum irisin levels and other lipid parameters, BMI, ECHO findings, CHA2DS2-VASc score or EAT thickness. The effects on serum irisin levels of heart failure, hypertension, stroke and basic demographic characteristics, such as sex, were assessed separately, and no significant effect of these parameters on serum irisin levels was observed.

\section{DISCUSSION}

No statistically significant difference was determined in our study between serum irisin levels during acute AF rhythm and at $24 \mathrm{~h}$ and $72 \mathrm{~h}$ after restoration of sinus rhythm via CV. Significant negative cor- 
relation was determined only between patients' basal serum irisin levels and the lipid parameter LDL. No significant correlation was determined between basal serum irisin levels and other lipid parameters, BMI, ECHO findings, CHADVASC2 score or EAT thickness. Ours is the first study to investigate the relation between $\mathrm{AF}$ and irisin.

Irisin is released by heart and skeletal muscle, the liver and kidney, the peripheral nerve sheath, and the dermis and hypodermis (12). Heart muscle cells produce more irisin than skeletal muscles (13). Irisin has been identified as a thermogenic protein responsible for energy consumption by converting white adipose tissue into brown adipose tissue. It activates the thermogenic function by increasing uncoupling protein-1 (UCP-1) expression in subcutaneous adipose tissue. Increased UCP1 results in heat production by reducing ATP synthesis, leading to greater consumption of energy resources $(10,11)$. EAT, a brown adipose tissue found between the epicardium and visceral layers of the pericardium, possesses high levels of UCP-1 and protects the myocardium against hypothermia by releasing fatty acid. Studies have shown that EAT thickness is correlated with the severity and incidence of AF $(14,15)$. Inflammatory cytokines released from EAT are thought to contribute to the persistence of $\operatorname{AF}(10,12,16)$. In addition, studies of the relation between irisin levels and myocardial infarction have determined that low irisin levels play a role in protecting myocardial cells against energy loss $(12,17)$.

In their experimental study, Kuloglu et al. determined low irisin levels in the first 24 $\mathrm{h}$ of myocardial infarction (12). Aydin et al. observed a gradual decrease up to $48 \mathrm{~h}$ in saliva and serum irisin levels in acute myocardial infarction patients, and reported that levels began rising after $72 \mathrm{~h}$ (17). While it is possible that serum irisin levels decrease gradually for the first $48 \mathrm{~h}$ following $\mathrm{CV}$ and establishment of sinus rhythm in $\mathrm{AF}$, and that they subsequently begin increasing, through the same mechanism, after $72 \mathrm{~h}$, our findings do not support this hypothesis. We determined no significant change in irisin levels at $24 \mathrm{~h}$ and $72 \mathrm{~h}$ post-CV.

Sahin et al. determined significantly higher serum irisin levels and EAT thickness in hyperthyroid patients compared to a control group, and that EAT was positively and independently correlated with serum irisin. Those authors reported that metabolic
Table 1. Baseline characteristics of acute AF patients

\begin{tabular}{ll}
\hline Variables & Acute AF $(\mathbf{n}=\mathbf{3 1})$ \\
\hline Age (years); mean \pm SD & $59.1 \pm 10.9$ \\
\hline Gender; $\mathrm{n}(\%)$ & \\
\hline Female & $17(54.8 \%)$ \\
Male & $14(45.2 \%)$ \\
\hline BMI $\left(\mathrm{kg} / \mathrm{m}^{2}\right) ;$ mean $\pm \mathrm{SD}$ & $31.35 \pm 6.3$ \\
\hline Comorbid diseases; $\mathrm{n}(\%)$ & \\
\hline Hyperlipidemia & $6(19.4 \%)$ \\
Coronary artery disease & $6(19.4 \%)$ \\
Hypertension & $17(54.8 \%)$ \\
Diabetes mellitus & $0(0 \%)$ \\
Smoking & $4(12.9 \%)$ \\
Stroke/TIA history & $1(3.2 \%)$ \\
Heart failure & $2(6.5 \%)$ \\
\hline CHA2DS2-VASc score; mean $\pm \mathrm{SD}$ & $1.71 \pm 1.37$ \\
\hline
\end{tabular}

ECHO findings; mean \pm SD

LVEF (\%)

$63 \pm 3.7$

$\operatorname{LVESD}(\mathrm{mm})$

$31.4 \pm 4.5$

LVEDD $(\mathrm{mm})$

$47.8 \pm 5.1$

LA diameter $(\mathrm{mm})$

$40.1 \pm 5.3$

IVS thicknesses (mm)

$11.1 \pm 1.5$

PW thicknesses (mm)

$10.4 \pm 1.3$

$\mathrm{RV}$ diameter $(\mathrm{mm})$

$33.9 \pm 3.6$

RA diameter (mm)

$39.7 \pm 4.7$

sPAP

$26.9 \pm 8.9$

Epicardial fat thickness ( $\mathrm{mm})$

$2.72 \pm 0.89$

Cardioversion type; $\mathrm{n}(\%)$

Medical 14(45.2\%)

Electrical 17(54.8\%)

AF:Atrial fibrillation, BMI:Body mass index, ECHO:Echocardiography, IVS:Interventricular septum, LA:Left atrium, LVEDD:Left ventricular end-diastolic diameter, LVEF: Left ventricular ejection fraction, LVESD:Left ventricular end-systolic diameter, PW:Posterior wall, RA:Right atrium, RV:Right ventricle, SD:Standard deviation, sPAP:Pulmonary artery pressure, TIA:Transient ischemic attack

Table 2. Patients' basal laboratory findings and serum irisin levels

\begin{tabular}{ll}
\hline Variables & Acute AF $(\mathbf{n}=\mathbf{3 1})$ \\
\hline Laboratory findings; & \\
\hline Glucose $(\mathrm{mg} / \mathrm{dL})$ & $110.7 \pm 37.7$ \\
Creatinine $(\mathrm{mg} / \mathrm{dL})$ & $0.84 \pm 0.22$ \\
TC $(\mathrm{mg} / \mathrm{dL})$ & $189.2 \pm 48.9$ \\
TG $(\mathrm{mg} / \mathrm{dL})$ & $129.3 \pm 55.9$ \\
LDL-c $(\mathrm{mg} / \mathrm{dL})$ & $119.1 \pm 41.2$ \\
HDL-c $(\mathrm{mg} / \mathrm{dL})$ & $43.1 \pm 11.2$ \\
Hemoglobin $(\mathrm{g} / \mathrm{dL})$ & $13.6 \pm 2$ \\
WBC $(\mathrm{U} / \mathrm{L})$ & $8370.3 \pm 3518.1$ \\
Platelet $(\mathrm{U} / \mathrm{L})$ & $244093.7 \pm 74606.6$ \\
TSH $(\mu \mathrm{IU} / \mathrm{mL})$ & $1.4 \pm 1.49$ \\
fT4 $(\mathrm{ng} / \mathrm{dL})$ & $4.2 \pm 16.9$ \\
CRP $(\mathrm{mg} / \mathrm{dL})$ & $3.08 \pm 7.09$ \\
\hline Irisin levels $(\mu \mathrm{g} / \mathrm{mL}) ;$ & \\
\hline Initial & $7.68 \pm 2.96 \mathrm{a}$ \\
24-h & $7.52 \pm 2.56 \mathrm{~b}$ \\
$72-\mathrm{h}$ & $7.71 \pm 2.63 \mathrm{c}$ \\
\hline
\end{tabular}

$p$ value between $a$ and $b=0.734$, $p$ value between $a$ and $c=0.958$, $p$ value between $b$ and $\mathrm{c}=0.643$. AF:Atrial fibrillation, CRP:C-reactive protein, fT4:Free thyroxine, HDL-c:High- 
density lipoprotein cholesterol, LDL-c:Low-density lipoprotein cholesterol, TC:Total cholesterol, TG:Triglycerides, TSH:Thyroid-stimulating hormone, WBC:White blood cell

Table 3. Correlations between patients' basal serum irisin levels and clinical characteristics, ECHO findings and laboratory findings

\begin{tabular}{lll}
\hline Variables & r values & p values \\
\hline Epicardial fat thickness & 0.089 & 0.627 \\
\hline CHA2DS2-VASc score & -0.162 & 0.375 \\
\hline BMI & 0.099 & 0.591 \\
\hline LA diameter & -0.017 & 0.926 \\
\hline LVESD & 0.224 & 0.218 \\
\hline LVEDD & 0.150 & 0.413 \\
\hline IVS thicknesses & 0.067 & 0.717 \\
\hline PW thicknesses & -0.047 & 0.799 \\
\hline LVEF & 0.146 & 0.425 \\
\hline RA diameter & -0.024 & 0.897 \\
\hline RV diameter & -0.036 & 0.844 \\
\hline sPAP & -0.263 & 0.146 \\
\hline Glucose & 0.212 & 0.243 \\
\hline Creatinine & 0.017 & 0.925 \\
\hline TC & -0.430 & 0.014 \\
\hline TG & -0.070 & 0.703 \\
\hline LDL-c & -0.519 & 0.002 \\
\hline HDL-c & -0.028 & 0.880 \\
\hline TSH & -0.053 & 0.773 \\
\hline CRP & 0.070 & 0.704 \\
\hline Hemoglobin & -0.126 & 0.493 \\
\hline Platelet & -0.017 & 0.925 \\
\hline & -0.102 & 0.579 \\
\hline
\end{tabular}

BMI:Body mass index, CRP:C-reactive protein, ECHO: echocardiography, fT4:Free thyroxine, HDL-c:High-density lipoprotein cholesterol, IVS:Interventricular septum, LA:Left atrium, LDL-c:Low-density lipoprotein cholesterol, LVEDD:Left ventricular enddiastolic diameter, LVEF: Left ventricular ejection fraction, LVESD:Left ventricular endsystolic diameter, PW:Posterior wall, RA:Right atrium, RV:Right ventricle, SD:Standard deviation, sPAP:Pulmonary artery pressure, TC:Total cholesterol, TG:Triglycerides, TSH:Thyroid-stimulating hormone, WBC:White blood cell

disorders such as increased basal metabolic rate and thermogenesis in hyperthyroid status might be indirectly associated with an increase in EAT and serum irisin levels (18). However, our findings are not compatible with those of Sahin et al. Although our patient group also consisted of acute AF patients with a high basal metabolic positive correlation between irisin levels and EAT thickness, while we observed no significant correlation. These findings indicate that further studies are now needed to investigate the relation between EAT thickness and serum irisin levels.

We observed no correlation between BMI and serum irisin levels in the present study. Although Sahin et al. and Kaneda et al.s results support our finding, other studies have reported a positive correlation between BMI and irisin levels $(10,20)$, while Moreno-Navarette et al. determined negative correlation (21). Although we determined significant negative correlation only between basal irisin levels and the lipid parameter LDL, we observed no significant correlation with total cholesterol, triglyceride or HDL. Sahin et al. and Kaneda et al. reported no correlation between serum irisin and any lipid parameter, although other studies have reported positive correlation between irisin and LDL and total cholesterol (22). Based on these findings, it is impossible to draw any definite conclusion concerning the relation between serum irisin and BMI and lipid parameters.

\section{LIMITATIONS}

The main limitation to our study is the relatively low number of patients enrolled. The second limitation is that the data could not be compared against a healthy control group. Additionally, serum irisin values were not investigated $48 \mathrm{~h}$ after CV.

\section{CONCLUSION}

We determined no change in acute AF patients' basal irisin levels and those at $24 \mathrm{~h}$ and $72 \mathrm{~h}$ following restoration of normal sinus rhythm after $\mathrm{CV}$ procedures. In addition, our findings revealed no correlation between AF patients' EAT thickness and serum irisin levels. More extensive studies are now needed for a better understanding of the association between serum irisin levels and AF and EAT.

\section{REFERENCES}

1. Kirchhof P, Benussi S, Kotecha D, Ahlsson A, Atar D, Casadei B, et al. 2016 ESC Guidelines for the management of atrial fibrillation developed in collaboration with EACTS. Eur J Cardiothorac Surg 2016;50(5):e1-e88.

2. Barbey O, Pierre S, Duran MJ, Sennoune S, Levy S, Maixent JM. Specific up-regulation of mitochondrial F0F1-ATPase activity after short episodes of atrial fibrillation in sheep. J Cardiovasc Electrophysiol $2000 ; 11(4): 432-8$. 
3. White CW, Kerber RE, Weiss HR, Marcus ML. The effects of atrial fibrillation on atrial pressure-volume and flow relationships. Circ Res $1982 ; 51(2): 205-15$

4. Ausma J, Coumans WA, Duimel H, Van der Vusse GJ, Allessie MA, Borgers. Atrial high energy phosphate content and mitochondrial enzyme activity during chronic atrial fibrillation. Cardiovasc Res 2000;47(4):788-96

5. Leistad E, Aksnes G, Verburg E, Christensen G. Atrial contractile dysfunction after short-term atrial fibrillation is reduced by verapamil but increased by BAY K8644. Circulation 1996;93(9):1747-54

6. Cha YM, Dzeja PP, Shen WK, Jahangir A, Hart CY, Terzic A, et al. Failing atrial myocardium: energetic deficits accompany structural remodeling and electrical instability. Am J Physiol Heart Circ Physiol 2003;284(4):H1313-20.

7. Seppet E, Eimre M, Peet N, Paju K, Orlova E, Ress M, et al. Compartmentation of energy metabolism in atrial myocardium of patients undergoing cardiac surgery. Mol Cell Biochem.2005; 270(1-2):49-61

8. Mihm MJ, Yu F, Carnes CA, Reiser PJ, McCarthy PM, Van Wagoner DR, et al. Impaired myofibrillar energetics and oxidative injury during human atrial fibrillation. Circulation. 2001;104(2):174-80.

9. Tsuboi M, Hisatome I, Morisaki T, Tanaka M, Tomikura Y, Takeda S, et al. Mitochondrial DNA deletion associated with the reduction of adenine nucleotides in human atrium and atrial fibrillation. Eur J Clin Invest 2001;31(6):489-96.

10. Aydin S. Three new players in energy regulation: preptin, adropin and irisin. Peptides. 2014;56:94-110.

11. Bostrom P, Wu J, Jedrychowski MP, Korde A, Ye L, Lo JC, et al. A PGC1-alpha-dependent myokine that drives brown-fat-like development of white fat and thermogenesis. Nature 2012;481(7382):463-8.

12. Kuloglu T, Aydin S, Eren MN, Yilmaz M, Sahin I, Kalayci M, et al. Irisin: a potentially candidate marker for myocardial infarction. Peptides 2014;55:85-91.

13. Park SE, Park CY, Sweeney G. Biomarkers of insulin sensitivity and insulin resistance: Past, present and future. Crit Rev Clin Lab Sci 2015;52:180-90.

14. Shin SY, Yong HS, Lim HE, Na JO, Choi CU, Choi JI, et al. Total and interatrial epicardial adipose tissues are independently associated with left atrial remodeling in patients with atrial fibrillation. J Cardiovasc Electrophysiol 2011;22:647-655

15. Tsao HM, Hu WC, Wu MH, Tai CT, Lin YJ, Chang SL, et al. Quantitative analysis of quantity and distribution of epicardial adipose tissue surrounding the left atrium in patients with atrial fibrillation and effect of recurrence after ablation. Am J Cardiol 2011;107:1498-1503.

16. Hatem SN, Sanders P. Epicardial adipose tissue and atrial fibrillation. Cardiovasc Res 2014;102(2):205-13.

17. Aydin S, Aydin S, Kobat MA, Kalayci M, Eren MN, Yilmaz M, et al. Decreased saliva/serum irisin concentrations in the acute myocardial infarction promising for being a new candidate biomarker for diagnosis of this pathology. Peptides 2014;56:141-5.

18. Sahin M, Canpolat AG, Corapcioglu D, Canpolat U, Emral R, Uysal AR. Association between circulating irisin levels and epicardial fat in patients with treatment-naïve overt hyperthyroidism. Biomarkers $2018 ; 25: 1-6$.

19. Kaneda H, Nakajima T, Haruyama A, Shibasaki I, Hasegawa T, Sawaguchi T, et al. Association of serum concentrations of irisin and theadipokines adiponectin and leptin with epicardial fat in cardiovascular surgery patients. PLoS One. 2018;13(8):e0201499

20. Stengel A, Hofmann T, Goebel-Stengel M, Elbelt U, Kobelt P, Klapp BF. Circulating levels of irisin in patients with anorexia nervosa and different stages of obesity - correlation with body mass index. Peptides 2013;39:125-30

21. Moreno-Navarette JM, Ortega F, Serrano M, Guerra E, Pardo G, Tinahones F, et al. Irisin is expressed and produced by human muscle and adipose tissue in association with obesity and insulin resistance. J Clin Endocrinol Metab 2013;98:E769-78.

22. Liu JJ, Wong MD, Toy WC. Lower circulating irisin is associated with type 2 diabetes mellitus. J Diabetes Complications 2013;27:36569 